\title{
NLO QCD calculations with the OPP method
}

\section{A. van Hameren}

The H. Niewodniczański Institute of Nuclear Physics

Polisch Academy of Sciences

Radzikowskiego 152, 31-342 Krakow, Poland

E-mail: hameren@ifj.edu.pl

\section{C.G. Papadopoulos*}

Institute of Nuclear Physics,

NCSR Demokritos,

15310 Athens, Greece

E-mail: costas.papadopoulosecern.ch

\section{R. Pittau}

Departamento de Física Teórica y del Cosmos

Centro Andaluz de Física de Partículas Elementales (CAFPE)

Universidad de Granada, E-18071 Granada, Spain

E-mail: pittau@ugr.es

An algorithm to compute automatically NLO QCD corrections for all momentum, color, and helicity configurations of the external particles is presented. It has been implemented in new package HELAC-1L, using HELAC for the evaluation of tree-level off-shell sub-amplitudes, CutTools for OPP-reduction and OneLOop for the evaluation of one-loop scalar integrals. Some recent results obtained with this package are presented.

European Physical Society Europhysics Conference on High Energy Physics, EPS-HEP 2009,

July 16 - 222009

Krakow, Poland

This work was partially supported by RTN European Programme, MRTN-CT-2006-035505 (HEPTOOLS, Tools and Precision Calculations for Physics Discoveries at Colliders).

*Speaker. 


\section{Introduction}

In order to deal with the data from the experiments at LHC for the study of elementary particles, signals and potential backgrounds for new physics have to be under control at sufficient accuracy [1]. In particular, hard processes with high multiplicities, involving many particles or partons, cannot be neglected. On top of that, such processes have to be dealt with at the next-toleading order (NLO) level to, for example, reduce the scale dependence of observables and to have a better description of the shape of their distributions.

At the leading order in perturbation theory, many tools are already available that are able to simulate any scattering process involving up to several partons [2]. These tools are highly automated and they have been widely used [3]. At the next-to-leading order the situation is currently less advanced. MCFM [4] is able to produce results at NLO accuracy for specific scattering processes, based on analytic calculations. Regarding the calculation of one-loop amplitudes, the only automatic tool available for some time now was FeynCalc [5] and FormCalc [6]. These tools rely heavily on the use of computer algebra. For processes with two particles in the final state, their performance is very satisfactory. There exist several important calculations that make use of these automatic packages and FeynArts [7], QGRAF [8], producing results with up to four particles in the final state [9], but for the moment no publicly available automatic tool exists. Recently a program called GOLEM [10] has been presented, that is able to deal with processes up to six external legs. It will also provide an alternative to compute automatically one-loop amplitudes [11].

The aforementioned programs express one-loop amplitudes in Feynman graphs, which again are expressed in terms of one-loop tensor integrals. These are then calculated using universal reduction techniques, independent of the amplitude, by expressing them in terms of scalar integrals. In a very different line of thinking, starting from the pioneering work in [12], a new approach has been set forward, known under the name of unitarity approach, which has been proven very powerful in computing multi-parton amplitudes in QCD that seemed to be impossible with the traditional Feynman graph approach. The reason is that one-loop amplitudes are calculated by using tree-order building blocks, that are either known analytically with very compact expressions, or can be evaluated using fast recursive equations. Nevertheless a systematic framework to develop a generic computation of any one-loop amplitude was missing, limiting the applicability of the method.

Using the crucial input from [13], this problem has been first solved in [14], introducing a systematic framework in order to calculate all coefficients of the scalar integrals, as well as part of the so-called rational contribution, related to the occurrence of UV-divergences. The remaining rational part can be reproduced by counter terms encoded in tree-like Feynman rules involving up to four fields [15]. Therefore, this method, known as the OPP method, provides a self-contained framework for the evaluation of the full one-loop amplitude. In [16], the OPP method was applied within the so-called generalized unitarity approach $[13,17]$ in order to get also the full rational contribution to the amplitude, paying the price to work with tree-amplitudes in higher dimensions.

The systematic extraction of all coefficients and of the rational term opened the road for the construction of tools that are able to compute one-loop amplitudes with any number of particles. BlackHat [18] and Rocket [19] were the first tools to realize such a possibility. In the following, we report on the development of a new program. 


\section{HELAC-1L}

For any one-loop amplitude, a number $n$ of propagator denominators can be identified such that the amplitude can be represented in the form

$$
\mathscr{A}=\sum_{I \subset\{1, \ldots, n\}} \int \frac{\mu^{4-d} d^{d} \bar{q}}{(2 \pi)^{d}} \frac{\bar{N}_{I}(\bar{q})}{\prod_{i \in I} \bar{D}_{i}(\bar{q})} \quad, \quad \bar{D}_{i}(\bar{q})=\left(\bar{q}+p_{i}\right)^{2}-m_{i}^{2},
$$

where the loop momentum $\bar{q}$ and the numerator polynomials $\bar{N}_{I}(\bar{q})$ are considered to be evaluated in $d$ dimensions. The $p_{i}$ are combinations of the external momenta, and the $m_{i}$ are the masses of particles running a loop. It is well-known that for $d \rightarrow 4$, each such term can be cast into the form

$$
\int \frac{\mu^{4-d} d^{d} \bar{q}}{(2 \pi)^{d}} \frac{\bar{N}_{I}(\bar{q})}{\prod_{i \in I} \bar{D}_{i}(\bar{q})}=\sum_{j} d_{j} \operatorname{Box}_{j}+\sum_{j} c_{j} \operatorname{Tri}_{j}+\sum_{j} b_{j} \operatorname{Bub}_{j}+\sum_{j} a_{j} \operatorname{Tad}_{j}+R
$$

where Box, Tri, Bub and Tad refer to the well known one-loop scalar integrals and $R=R_{1}+R_{2}$ is the rational term. Given the momenta $p_{i}$, the masses $m_{i}$ and a function evaluating the 4-dimensional numerator $N_{I}$ as function of $q$, the program CutTools [20] identifies the scalar integrals to be evaluated, calculates the coefficients, and determines $R_{1}$ following the OPP method. The scalar integrals can be evaluated with the tools from [21] or with our own code OneLOop.

For the evaluation of the numerator, the program HELAC [22] is used. It efficiently calculates tree-level amplitudes by applying recursive relations for off-shell currents [23]. The convenience of its applicability stems from the freedom in the choice of the initial decomposition represented by the summation in Eq. (2.1). In this application it is a sum over all topologically inequivalent partitions of the external particles. A few examples are depicted on the right, each graph corresponds to an instance of the label $I$ in Eq. (2.1). The external particles are labelled by powers of 2 , and the blobs do not contain propagators depending on the integration momentum $q$. HELAC can easily evaluate such a term by considering one line to be cut, and summing over all possible internal spin, flavor and/or color states of that cut line. Each term in this sum is an amplitude with two more external particles, restricted such that it only
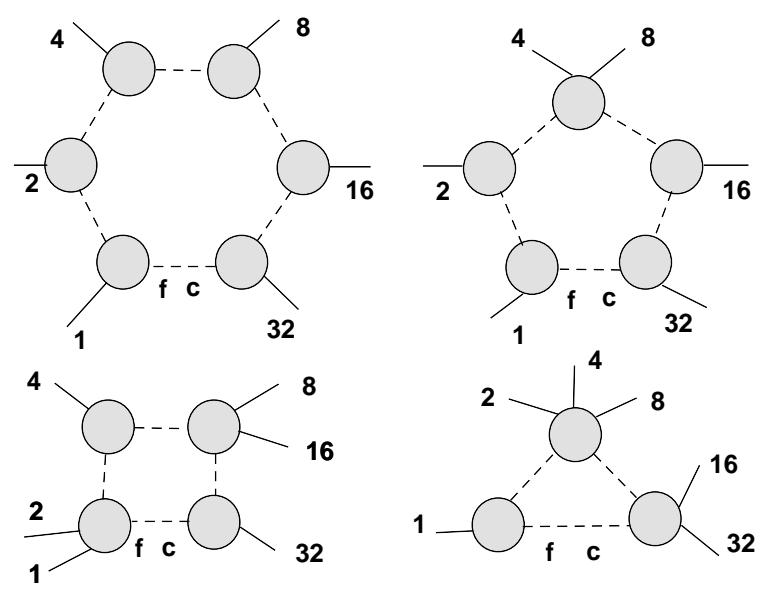
contains Feynman graphs including all the propagators eventually forming the loop. This can easily be accomplished by putting together the necessary off-shell currents provided by HELAC.

Regarding the color-treatment, HELAC uses the color-connection representation. External quarks and anti-quarks are represented by a color or anti-color index, gluons are represented by a pair of color/anti-color indices, and HELAC calculates all non-zero tree-level color-connections. The same is done to for the amplitudes with two more particles necessary to calculate the one-loop numerators. The contributions from non-planar loops are obtained by taking into account different orderings of these extra particles among the other external particles.

The evaluation of the rational part $R_{2}$, finally, follows the same line as the calculation of the counter terms necessary for renormalization, and is comparably trivial. 


\section{RESULTS}

The current implementation allows to calculate any one-loop virtual matrix element, for all color and helicity configurations, with any external particle and with particles in the loop that can be either gluons (ghosts) or quarks of any flavor. Moreover the cut-constructible part can be obtained for any internal particle. When the rational counterterms for the full Standard Model will be implemented, then any one-loop amplitude will be obtainable. The calculation is done in a fully automatic way, and it is purely numerical.

To illustrate the capabilities of the developed package, we present here results for one of the most complicated processes, $p p \rightarrow t \bar{t}+2$ jets, for a given phase-space point. For details of the calculations we refer to [24].

\begin{tabular}{|l|c|c|c|}
\hline \multicolumn{5}{|c|}{$p p \rightarrow t \bar{t}+2$ jets } \\
\hline \hline \multicolumn{5}{|c|}{$u \bar{u} \rightarrow t \bar{t} g g$} \\
\hline LO: $3.534870065372714 \mathrm{E}-06$ \\
\hline \multicolumn{5}{|c|}{$g g \rightarrow t \bar{t} g g$} \\
\hline HELAC-1L & $-6.127108113312741 \mathrm{E}-05$ & $-1.874963444741646 \mathrm{E}-04$ & $-3.305349683690902 \mathrm{E}-04$ \\
\hline$I(\varepsilon)$ & $-6.127108113312702 \mathrm{E}-05$ & $-1.874963445081074 \mathrm{E}-04$ & $\varepsilon^{0}$ \\
\hline \multicolumn{5}{|c|}{ LO: $1.599494381233976 \mathrm{E}-05$} \\
\hline HELAC-1L & $-3.838786514961561 \mathrm{E}-04$ & $-9.761168899507888 \mathrm{E}-04$ & $-5.225385984750410 \mathrm{E}-04$ \\
\hline$I(\varepsilon)$ & $-3.838786514961539 \mathrm{E}-04$ & $-9.761168898436521 \mathrm{E}-04$ & \\
\hline
\end{tabular}

The momenta used to obtain the above result are

\begin{tabular}{l|rrrr} 
& $p_{x}$ & $p_{y}$ & $p_{z}$ & $E$ \\
\hline$u(g)$ & 0 & 0 & 250 & 250 \\
$\bar{u}(g)$ & 0 & 0 & -250 & 250 \\
$t$ & 12.99421901255723 & -9.591511769543683 & 75.05543670827210 & 190.1845561691092 \\
$\bar{t}$ & 53.73271578143694 & -0.2854146459513714 & 17.68101382654795 & 182.9642163285034 \\
$g$ & -41.57664370692741 & 3.895531135098977 & -91.94931862397770 & 100.9874727883170 \\
$g$ & -25.15029108706678 & 5.981395280396083 & -0.7871319108423604 & 25.86375471407044
\end{tabular}

Further results can be found in [24]. Moreover results concerning a realistic cross section calculation for $p p \rightarrow t \bar{t} b \bar{b}$ can be found in [25]. 


\section{References}

[1] Z. Bern et al. [NLO Multileg Working Group], arXiv:0803.0494 [hep-ph].

[2] M. L. Mangano, M. Moretti, F. Piccinini, R. Pittau and A. D. Polosa, JHEP 0307 (2003) 001. F. Maltoni and T. Stelzer, JHEP 0302 (2003) 027.

T. Gleisberg, S. Hoche, F. Krauss, A. Schalicke, S. Schumann, J. C. Winter, JHEP 0402 (2004) 056.

A. Kanaki and C. G. Papadopoulos, arXiv:hep-ph/0012004.

C. G. Papadopoulos, Comput. Phys. Commun. 137 (2001) 247.

A. Cafarella, C. G. Papadopoulos and M. Worek, arXiv:0710.2427 [hep-ph].

W. Kilian, T. Ohl and J. Reuter, arXiv:0708.4233 [hep-ph].

[3] J. Alwall et al., Eur. Phys. J. C 53 (2008) 473.

[4] R. K. Ellis, Nucl. Phys. Proc. Suppl. 160 (2006) 170.

[5] R. Mertig, M. Bohm and A. Denner, Comput. Phys. Commun. 64 (1991) 345.

[6] T. Hahn, arXiv:0901.1528 [hep-ph].

[7] T. Hahn, Comput. Phys. Commun. 140 (2001) 418.

[8] P. Nogueira, J. Comput. Phys. 105 (1993) 279.

[9] W. Beenakker, S. Dittmaier, M. Kramer, B. Plumper, M. Spira, P. Zerwas, Nucl.Phys.B653(2003)151.

A. Denner, S. Dittmaier, M. Roth and L. H. Wieders, Phys. Lett. B 612 (2005) 223.

S. Dittmaier, P. Uwer and S. Weinzierl, Phys. Rev. Lett. 98 (2007) 262002.

S. Dittmaier, S. Kallweit and P. Uwer, Phys. Rev. Lett. 100 (2008) 062003.

A. Bredenstein, A. Denner, S. Dittmaier and S. Pozzorini, JHEP 0808 (2008) 108.

[10] T. Binoth, J. P. Guillet, G. Heinrich, E. Pilon and T. Reiter, arXiv:0810.0992 [hep-ph].

[11] T. Reiter, arXiv:0903.0947 [hep-ph].

[12] Z. Bern, L. J. Dixon, D. C. Dunbar and D. A. Kosower, Nucl. Phys. B 435 (1995) 59.

Z. Bern, L. J. Dixon, D. C. Dunbar and D. A. Kosower, Nucl. Phys. B 425 (1994) 217.

[13] R. Britto, F. Cachazo and B. Feng, Nucl. Phys. B 725 (2005) 275.

[14] G. Ossola, C. G. Papadopoulos and R. Pittau, Nucl. Phys. B 763 (2007) 147.

G. Ossola, C. G. Papadopoulos and R. Pittau, JHEP 0707 (2007) 085.

[15] G. Ossola, C. G. Papadopoulos and R. Pittau, JHEP 0805 (2008) 004.

P. Draggiotis, M. V. Garzelli, C. G. Papadopoulos and R. Pittau, JHEP 0904 (2009) 072.

[16] W. T. Giele, Z. Kunszt and K. Melnikov, JHEP 0804 (2008) 049.

R. K. Ellis, W. T. Giele, Z. Kunszt and K. Melnikov, arXiv:0806.3467 [hep-ph].

A. Lazopoulos, arXiv:0812.2998 [hep-ph].

[17] C. Anastasiou, R. Britto, B. Feng, Z. Kunszt and P. Mastrolia, Phys. Lett. B 645 (2007) 213.

[18] C. F. Berger et al., Phys. Rev. D 78 (2008) 036003.

[19] W. T. Giele and G. Zanderighi, JHEP 0806 (2008) 038.

[20] G. Ossola, C. G. Papadopoulos and R. Pittau, JHEP 0803 (2008) 042.

[21] G. J. van Oldenborgh, Comput. Phys. Commun. 66 (1991) 1.

R. K. Ellis and G. Zanderighi, JHEP 0802 (2008) 002.

[22] A. Kanaki and C. G. Papadopoulos, Comput. Phys. Commun. 132 (2000) 306. 
[23] F. A. Berends and W. T. Giele, Nucl. Phys. B 306 (1988) 759.

F. Caravaglios and M. Moretti, Phys. Lett. B 358 (1995) 332.

P. Draggiotis, R. H. P. Kleiss and C. G. Papadopoulos, Phys. Lett. B 439 (1998) 157.

[24] A. van Hameren, C. G. Papadopoulos and R. Pittau, arXiv:0903.4665 [hep-ph].

[25] G. Bevilacqua, M. Czakon, C. G. Papadopoulos, R. Pittau and M. Worek, arXiv:0907.4723 [hep-ph]. 